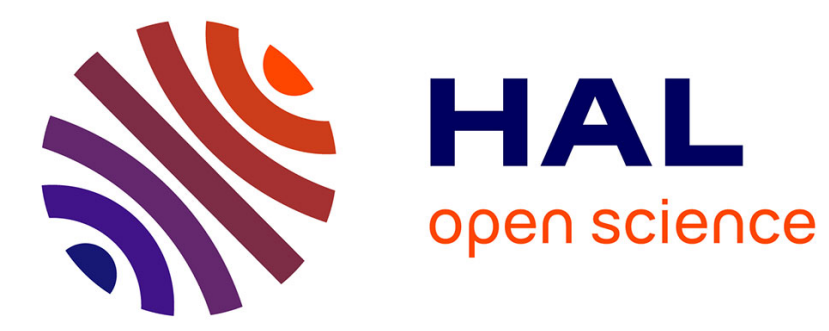

\title{
L'Ethnologue dans le showbiz. Apprendre à parler métier
}

Eliane Daphy

\section{To cite this version:}

Eliane Daphy. L'Ethnologue dans le showbiz. Apprendre à parler métier. Journal des anthropologues, 1994, 57-58 ("L'Anthropologue face à la langue", coordonné par Diana Rey-Hulman et Galina Kabakova, et "co, pp.29-39. halshs-00009912

\section{HAL Id: halshs-00009912 https://shs.hal.science/halshs-00009912}

Submitted on 3 Apr 2006

HAL is a multi-disciplinary open access archive for the deposit and dissemination of scientific research documents, whether they are published or not. The documents may come from teaching and research institutions in France or abroad, or from public or private research centers.
L'archive ouverte pluridisciplinaire HAL, est destinée au dépôt et à la diffusion de documents scientifiques de niveau recherche, publiés ou non, émanant des établissements d'enseignement et de recherche français ou étrangers, des laboratoires publics ou privés. 


\title{
L’ETHNOLOGUE DANS LE SHOWBIZ
}

\author{
Apprendre à parler métier \\ Eliane DAPHY
}

(Laboratoire d'Anthropologie Urbaine ${ }^{*}$ )

\author{
Quand un vicomte \\ Rencontre un aut' vicomte \\ Qu'est ce qu'ils s'racontent \\ Des histoires de vicomtes... [...] \\ Quand un cul d'jatte \\ Rencontre un aut' cul d'jatte \\ Rien n'les épate \\ Qu'une histoir' de cul d'jatte ${ }^{1}$
}

Dans l'observation participante, il faut participer à la langue spécifique du groupe. Et la distance entre la langue de l'ethnologue et celle de ceux qu'il observe et écoute est parfois très grande : à lui de réduire la distance en apprenant cette autre langue qu'il croyait sienne, mais qui, pourtant, comme l'expérience lui a appris, ne lui permet pas de communiquer sur le terrain.

Mes recherches concernent le milieu des professionnels de la musique en France. Fidèle à la méthode ethnologique classique, j'obtiens mon matériel non seulement en observant des faits, mais aussi en écoutant des paroles: le regard et l'écoute se cumulent, parfois même jusqu'à se confondre. Mais, sur le terrain, bien sûr, je ne suis pas muette, et j'obtiens aussi mes informations en parlant, en échangeant des mots dans des relations interpersonnelles. Pour mener à bien mes [29] recherches, j'ai dû

\footnotetext{
L.A.U., C.N.R.S., 27 rue Paul Bert, 94204 Ivry-sur-Seine Cedex.

1. Quand un vicomte, chanson interprétée par Maurice Chevalier, paroles de Jean Nohain, musique de Mireille, éditions Raoul Breton, 1935.
} 
Daphy Éliane, 1994, "L'ethnologue dans le showbiz. Apprendre à parler

métier ", Journal des anthropologues 57-58 (L'anthropologue face à la

langue, coordonné par Diana Rey-Hulman et Galina Kabakova) : 29-39.

peu à peu apprendre à comprendre, puis à parler la langue des musiciens : le parler métier.

En 1908, le folkloriste Van Gennep attirait l'attention sur les différences linguistiques internes à une même langue, et proposait de distinguer la langue ordinaire, commune à tous les membres d'une société globale, et les langues spéciales, utilisées par des groupes restreints (classes d'âge, sexe, activités spécialisées...). Selon cet auteur, «chez nous, les sociétés secondaires ou restreintes sont d'ordinaire constituées par des individus ayant la même activité économique et les langues spéciales prennent le caractère de simples terminologies. » (Van Gennep $1908: 3$ ).

En quoi consiste cette langue ? Comment l'ai-je apprise ? Pourquoi la nécessité d'apprendre à la parler s'est-elle imposée ? Ne me suffisait-il pas de la comprendre ? Au-delà, peut-on parler d'apprendre une langue (nouvelle, étrangère) et donc de bilinguisme, pour un jargon, un dialecte, un lexique technique, c'est-à-dire une langue spéciale de la langue commune ? Pour répondre à ces interrogations, il convient de considérer le double statut de la langue pour l'ethnologie. D'une part, la langue est matériel et objet de la recherche, lorsque l'ethnologue étudie les rapports entre la culture et la langue et les fonctions de la langue pour un groupe. D'autre part, sur le terrain, la langue est un outil de communication par lequel l'ethnologue entre en relation avec le groupe, il faut alors s'interroger sur le rôle de l'échange verbal dans la méthode (participation et distanciation). Poser ces questions dans le cadre spécifique d'une recherche sur la France, qui met en présence des interlocuteurs de même langue maternelle, c'est aussi considérer que le problème de relations à la langue est propre à la méthode, à la relation ethnologue/observés, et ceci quel qu'en soit le terrain.

\section{Comprendre : la langue matériel}

L'objet de mes recherches concerne le travail et ses représentations dans le secteur de la musique marchande dans une optique où la pensée et le langage sont partie prenante de l'analyse des rapports de production (Godelier 1984). La manière particulière dont sont dites les [30] choses par ceux du métier mais aussi par le reste de la société permet de mettre en 
Daphy Éliane, 1994, "L'ethnologue dans le showbiz. Apprendre à parler

métier ", Journal des anthropologues 57-58 (L'anthropologue face à la

langue, coordonné par Diana Rey-Hulman et Galina Kabakova) : 29-39.

évidence le jeu permanent d'opposition et de complémentarité entre les intérêts du groupe restreint et ceux de la société globale.

Par exemple, dans la langue commune, un musicien ne travaille pas, il joue. Ceux du métier n'utilisent pas le terme jouer pour désigner leurs activités professionnelles rémunérées, ils emploient le terme faire suivi d'un complément direct qui précise un lieu (faire l'Olympia), un artiste (faire Aznavour), ou un type de prestations (faire une séance, une télé, une radio). Ils tournent en dérision cette contradiction, comme dans cette blague :

C'est le fils d'un musicien, sa maîtresse l'interroge à l'école : «Et qu'est-ce qu'ils font tes parents, mon petit bonhomme ? " Et le gamin répond: «Mon père il est musicien et ma mère, c'est pareil, elle travaille pas. »

Pour l'analyse sémantique, la langue est un matériel, une information comme les autres qu'il faut collecter, par le relevé du vocabulaire spécialisé de la technique et des activités de production, pour constituer un lexique des termes et des expressions. Cette collecte s'avère fructueuse sur le terrain du showbiz: ainsi, je pus réunir rapidement plus d'une centaine de mots et d'expressions concernant le vocabulaire du son. Richesse qui n'a rien d'étonnant de la part d'individus pour qui le son est la matière qu'ils travaillent: "dans les langues de métier, la prolifération conceptuelle correspond à une attention plus soutenue envers les propriétés du réel, à un intérêt mieux en éveil pour les distinctions qu'on peut y introduire " (Lévi-Strauss $1962: 5$ ).

Toutes ces tâches de relevé de terminologie technique ne posent pas de problème linguistique particulier, elles sont même éventuellement réalisables par un ethnologue qui ne possèderait qu'une connaissance élémentaire de la langue du groupe, voire aucune connaissance. Je prendrais trois exemples, tirés d'un récent travail sur la sonorisation de spectacles ${ }^{2}$.

2. Recherche réalisée dans le cadre d'un appel d'offres du ministère de la recherche sur la modernisation des entreprises et des services. 
Daphy Éliane, 1994, "L'ethnologue dans le showbiz. Apprendre à parler métier ", Journal des anthropologues 57-58 (L'anthropologue face à la langue, coordonné par Diana Rey-Hulman et Galina Kabakova) : 29-39.

En Angleterre et en France, j'ai effectué des observations participantes de spectacles auprès d'équipes de techniciens anglophones ${ }^{3}$; ma connaissance de l'anglais est suffisante pour permettre le dialogue. [31]

Contrairement aux situations similaires auprès des techniciens français, où il m'est toujours difficile d'interroger directement sur l'emploi du vocabulaire (en particulier les métaphores et les idiotismes), la communication avec les interlocuteurs se trouvait facilitée par mon absence de maîtrise de la langue anglaise: les sonorisateurs anglophones manifestaient une patience remarquable, acceptant même de noter par écrit les termes d'un vocabulaire de tradition orale. Tandis qu'un technicien français répondrait à ce genre de demande: "Ca s'écrit comme ça se prononce... », "regarde dans le dico » ou " ça s'écrit pas, ça se dit $» \ldots$

Au Mexique, et bien que ne possédant que des notions rudimentaires d'espagnol, j'ai réalisé une rapide enquête auprès d'un vieux musicien jouant sur des ordinateurs (synthétiseurs et boites à rythmes) de la musique traditionnelle ranchera dans un bar de Cuernavaca ${ }^{4}$. Un collègue mexicain, avec lequel je parlais anglais, faisait office de traducteur. Je communiquais souvent directement avec le musicien en utilisant le vocabulaire international de la technique, formé principalement à partir de l'anglais; vocabulaire que mon collègue ignorait, et que le musicien utilisait pour parler de sa technique.

\section{Parler la langue outil}

Dans l'enquête, la langue a la fonction d'outil de communication avec les informateurs, et comme les exemples précédents l'ont montré, il est nécessaire de parler technique pour faire une enquête, ce qui ne veut pas dire pour autant parler métier.

C'est pourquoi sur le terrain, et plus précisément dans la position d'observation participante, s'est imposée à moi la nécessité d'apprendre à parler la langue des informateurs.

\footnotetext{
3. En particulier des représentations de l'opéra Carmen à Londres, et le spectacle de Pavarotti au Palais Omniports de Paris-Bercy.

4. Petite ville touristique au Sud de Mexico.
} 
Daphy Éliane, 1994, "L'ethnologue dans le showbiz. Apprendre à parler

métier », Journal des anthropologues 57-58 (L'anthropologue face à la

langue, coordonné par Diana Rey-Hulman et Galina Kabakova) : 29-39.

Je n'ai pas eu le choix: parler métier, partager la langue est une condition sine qua non, exigée pour rester sur le terrain. L'apprentissage du langage, suivant le chemin que montrait le terrain, consistait principalement à apprendre à rire. Les musiciens font un usage systématique de la parole «pour de rire »: blagues, anecdotes, moqueries, sobriquets, dérision, détournements, métaphores... Ils manifestent d'ailleurs une [32] grande fierté à propos de cette compétence langagière, qui selon eux, est une "caractéristique de ceux qui font le métier ".

Lors des premiers séjours sur le terrain, confrontée à la façon particulière de dire les choses, je ne comprenais guère ce que disaient entre eux les musiciens. Le problème de compréhension se posait également dans les entretiens. Voici en exemple un extrait d'un entretien avec un musicien qui fait le métier.

Bernard était un musicien aux multiples activités: 45 ans, contrebassiste et altiste, formation classique, enseignant (dans des conservatoires de musique et avec des élèves hors institutions), musicien dans des orchestres de jazz, parfois remplaçant dans des orchestres classiques, arrangeur pour un célèbre compositeur de musique de films, à l'occasion compositeur pour la publicité, accompagnateur de chanteurs de variétés ou de rock.

- Est-ce que vous vous considérez plutôt comme un musicien classique ou comme un musicien de jazz?

- Comme je vous l'ai dit, je fais le métier. Je ne dirais pas que je suis un musicien classique, ça non, pas vraiment... Enfin, bien sûr, je fais quelques remplacements à l'Opéra de temps en temps, pour rendre service à des copains, cela fait partie du métier. Mais de là à me considérer comme un musicien classique... Voyons, comment vous expliquer? Voyez-vous, je n'ai pas de problème pour m'accorder, ma femme a souvent l'occasion de me voir jouer à la maison, et surtout, surtout, le plus important pour bien répondre à votre question sur 'musicien classique', vous devez savoir que j'ai de très mauvaises relations avec mon concierge...

Et il était parti dans un grand rire auquel fit écho mon silence stupéfait. J'avais interprété ses propos comme un refus de répondre à ma question, et son rire comme une moquerie. Son attitude était d'autant plus étonnante 
Daphy Éliane, 1994, "L'ethnologue dans le showbiz. Apprendre à parler

métier », Journal des anthropologues 57-58 (L'anthropologue face à la

langue, coordonné par Diana Rey-Hulman et Galina Kabakova) : 29-39.

et incompréhensible que l'entretien s'était déroulé jusqu'à ce moment (selon mon point de vue) dans de bonnes conditions. Lorsque je lui avais expliqué le but de mon travail: "je suis étudiante en ethnologie et je souhaite étudier le métier de musicien», il avait manifesté un grand enthousiasme et proposé de devenir «mon informateur », terme qu'il avait employé de sa propre initiative, tout en me précisant [33] qu'il avait suivi quelques cours d'ethnomusicologie à la grande époque de l'université Vincennes, et qu'il avait trouvé cela passionnant. Sur le carnet de notes où j'ai l'habitude de griffonner rapidement en cours d'entretiens certains points qui me paraissent nécessiter une relance, j'avais écrit: " classique/jazz : concierge ??? »...

Bernard s'excusa gentiment, en me faisant remarquer que «si je ne comprenais pas ce qu'il me disait, c'était parce que je ne connaissais rien au métier ». Et ensuite, il avait suggéré " d'en rester là pour l'instant », de nous tutoyer et de venir le rejoindre le soir " aux environs de minuit » dans un club de jazz où il allait retrouver des copains, ce qui « me permettrait de franchir un premier pas dans mon étude du métier ». J'ai interprété ses propositions comme une tentative de séduction (et donc un dérapage incontrôlé visant à transformer le rapport ethnologue/informateur). Je refusais poliment, sous un prétexte quelconque, et notre relation s'arrêta là.

Provisoirement: quelques années plus tard, je croisais de nouveau Bernard dans un concert; après avoir échangé (en nous tutoyant...), suivant la coutume des réseaux, des nouvelles sur nos trajectoires respectives, il évoqua au bar à l'entracte, dans l'hilarité générale (moi incluse), l'époque héroïque où je débarquais pour étudier les musiciens dans la jungle du showbiz avec mon petit magnétophone et mon petit carnet de notes, et où je ne comprenais rien quand on parlait métier. Un musicien présent avait surenchérit : « elle a fait des progrès, elle comprend toujours pas, mais au moins, maintenant elle rigole... »

Laissant à l'ethnologue chevronné la tâche de relever l'accumulation d'erreurs du novice (ethnocentrisme et sexocentrisme ${ }^{5}$, projection et transfert) et d'en mesurer les paramètres et les effets (Devereux 1986), je

5. Précisons toutefois que dans le milieu des musiciens, presque entièrement masculin, il existe pour les femmes le rôle de la groupie (objet sexuel), définie comme « admiratrice au grand cœur » dans L'argot des musiciens. 
Daphy Éliane, 1994, "L'ethnologue dans le showbiz. Apprendre à parler

métier », Journal des anthropologues 57-58 (L'anthropologue face à la

langue, coordonné par Diana Rey-Hulman et Galina Kabakova) : 29-39.

voudrais essayer de tirer maintenant les leçons de cette conversation en situant les propos tenus par Bernard sur les musiciens classiques dans le contexte du parler métier. Le lecteur doit savoir que les trois éléments de l'argument (savoir s'accorder, jouer à la maison, relations avec son concierge), référent à certains thèmes des blagues de [34] métier célèbres qui stigmatisent les défauts typiques attribués aux musiciens classiques. En résumé, disent ces blagues, le musicien classique ne sait pas s'accorder, il ne travaille jamais son instrument chez lui, et il n'hésite pas à se faire remplacer par son concierge.

Par rapport à ces blagues, Bernard procédait donc par allusion. Ce mécanisme de sur-codage, qui n'utilise que des éléments partiels de récits facétieux très répandus dans la tradition orale du métier, est caractéristique du parler métier. La connivence entre les "ceux du métier » repose sur le décodage des éléments comiques.

Précisons aussi que ma question telle que je l'avais posée n'avait aucune signification pour un musicien professionnel. Ce n'est pas l'objet ici d'exposer en détails les caractéristiques du métier de musicien, travail qui a été en partie déjà fait (Daphy \& Raveyre 1988 et 1989); il suffit de préciser qu'il consiste à cumuler simultanément des activités polyvalentes, dans différents genres musicaux.

Le temps permet de mesurer la distance parcourue. Aujourd'hui, lorsque je rencontre un musicien, je n'ai plus ces problèmes, il m'est facile de rire des anecdotes qu'on me raconte, de faire les commentaires appropriés, de poser les questions en employant les expressions adéquates. Parler métier est un savoir langagier qui permet de converser facilement entre gens du métier, mais c'est en même temps un outil qui permet de juger de l'extériorité, de reconnaître, à partir du vocabulaire employé, si quelqu'un est du métier ou non.

\section{L'apprentissage du parler métier : un rite initiatique}

Je n'entrepris pas immédiatement une collecte systématique des faits de langage qui ne concernaient pas directement le vocabulaire technique (en particulier les blagues, les expressions et les jeux de mots). Je me contentais de noter laconiquement sur mon journal de terrain: après le 
Daphy Éliane, 1994, "L'ethnologue dans le showbiz. Apprendre à parler

métier », Journal des anthropologues 57-58 (L'anthropologue face à la

langue, coordonné par Diana Rey-Hulman et Galina Kabakova) : 29-39.

concert, soirée comique, ainsi que quelques précisions: le nom des narrateurs, les participants, le thème des blagues et anecdotes racontées ainsi qu'un résumé des structures narratives. Les conditions des observations participantes (une tournée de concerts ou un enregistrement de disque par exemple) ne laissant parfois que quelques rares heures de [35] sommeil quotidien, je n'ai donc pas toujours immédiatement exploité la totalité de mes notes. II en résulte que certaines parties de mon journal de terrain me sont actuellement incompréhensibles; en particulier, celles concernant la langue. Ce qui permet d'en souligner certaines caractéristiques du parler métier : l'inscription dans le temps et la mobilité.

II me faut préciser brièvement deux aspects déterminants dans mon attitude d'évitement (inconsciente) du comique: d'une part, je ne voyais vraiment pas ce que cela pouvait avoir de drôle; d'autre part, les blagues que je comprenais, portant en particulier sur des thèmes sexuels ou scatologiques, étaient loin de provoquer mon adhésion. Je les jugeais particulièrement sexistes ou infantiles, attitude sexocentriste dont il a bien fallu me départir par la suite de l'enquête. Confrontée à mon désir inconscient d'idéalisation de l'Autre, habituel me semble-t-il en début de terrain, j'ai laissé de côté certains aspects dérangeants. Mais au-delà des déterminismes personnels, il convient dans l'analyse de prendre en compte la fonction initiatique de la langue pour le groupe.

Accéder au terrain est une chose, y rester une autre qui exige d'être acceptée. Si le constat est banal, la situation n'en est pas moins difficile à vivre. Ceux du métier ne sont pas particulièrement gentils entre eux, la compétition est exacerbée mais elle ne peut être dite clairement: la dérision et l'ironie, seul mode d'expression autorisée, sont la norme de l'échange verbal. Ma situation d'ethnologue (étrangère au milieu) et ma demande explicite (observation participante donc une forme d'intégration) me mettaient dans une situation similaire à celle du néophyte. Mon point de vue était sans arrêt sollicité : " elle est bonne, hein, celle-là ? ». Situation d'examen, où je devais fournir une réponse appropriée. Toute erreur était immanquablement dénoncée à la vindicte collective : « eh, les mecs, notre ethnologue trouve ça drôle/pas drôle! ». Je me retrouvais ainsi renvoyée à une extériorité qui remettait en cause ma présence: "si tu ne comprends rien au métier, qu'est-ce que tu fous là ? "Toutes mes tentatives de 
Daphy Éliane, 1994, "L'ethnologue dans le showbiz. Apprendre à parler

métier », Journal des anthropologues 57-58 (L'anthropologue face à la

langue, coordonné par Diana Rey-Hulman et Galina Kabakova) : 29-39.

justifications (" je viens pour apprendre ») étaient vaines et les demandes d'explications recevaient systématiquement une fin de non recevoir. II n'est pas possible d'expliquer "ce qu'il y a de drôle ». Face au rire, il n'y a pas de position intermédiaire, c'est soit l'adhésion, soit l'exclusion. Pour accéder au rire, il me [36] fallait nécessairement faire l'apprentissage du langage, comme façon d'exprimer la culture, et cela ne peut se faire que par la pratique. Parler métier, procéder par allusions hermétiques aux étrangers, fait partie du savoir initiatique qui intègre au groupe ; il permet de montrer que l'on fait partie de la grande famille de ceux qui font le métier (Daphy \& Raveyre 1988).

La moquerie, la blague, l'expression idiomatique ont une fonction pédagogique: c'est une façon de montrer au débutant (à l'apprenti) l'impropriété de sa question ou de sa formulation dans une situation donnée. Prenons un exemple dans l'ouvrage sur l'argot des musiciens (paru en 1992, et donc un outil dont je ne disposais pas à l'époque où j'ai réalisé mes enquêtes). Au musicien débutant posant la question de la tonalité d'un morceau: "c'est en quel ton ? " ou "c'est en quoi ? », on répondra "t'casses pas la tête, suis mon pied" ou " en tergal»; les formules de la réponse peuvent varier à l'infini, il suffit qu'elles provoquent par leur absurdité le rire complice des pros et le malaise du débutant. L'ethnologue, comme le novice, apprend ainsi à savoir formuler les bonnes questions : quoi, comment, dans quels termes, dans quelles situations.

Lorsque le groupe rejette celui qui se propose d'entrer dans le cercle des narrateurs de blagues, la manière de lui refuser la parole est pratiquement toujours identique : " tu la tiens d'où, celle-là ? ", " tu la tiens du chef d'orchestre de l'harmonie municipale? » «tu l'as apprise au bal annuel des pompiers ? » ou " c'est le machiniste du théâtre de Bécon-lesBruyères qui te l'a racontée ? ". Ces commentaires ironiques, qui renvoient le postulant à l'origine pas pro de son récit, dénient le caractère opératoire de son savoir. Ainsi, le groupe signifie que la place réclamée par le narrateur dans le groupe des pros est usurpée, et renvoie le postulant à son statut d'amateur.

S'oppose au parler métier une sorte de "parler amateur" dont on trouve de nombreux exemples dans le Dictionnaire de l'argot des musiciens : 
Daphy Éliane, 1994, "L'ethnologue dans le showbiz. Apprendre à parler

métier ", Journal des anthropologues 57-58 (L'anthropologue face à la

langue, coordonné par Diana Rey-Hulman et Galina Kabakova) : 29-39.

- « au faire le bœuf des musiciens de métier, certains amateurs voulant afficher un hyper-professionnalisme ont substitué le plus vigoureux taper le bœuf, sur le mode de taper le blues » [37]

- "gratteuse ne fait partie que du vocabulaire des non-musiciens"

- « musico : terme qui a connu une telle fortune auprès des nonmusiciens que nous ne pouvons le passer sous silence. Et pourtant, les musiciens entre eux ne l'emploient que rarement, le terme étant ressenti comme vaguement péjoratif et faussement pro ».

Quelques rapides remarques, pour conclure provisoirement.

J'ai appris sur le terrain que la communication en langue commune ne permet jamais d'accéder à cette connaissance approfondie (de l'intérieur) de l'autre que l'ethnologie se donne comme objet. Elle permet tout au plus un simulacre d'échange, où questionneur et questionné s'accordant sur un vocabulaire commun, échangent des propos de dupes réciproques, et ceci est particulièrement vrai dans les entretiens.

L'acquisition du parler métier nécessaire à la compréhension et à la communication suit un processus d'accumulation longue, dont les mécanismes échappent en grande partie à la conscience. Une fois acquise cette compétence linguistique, garante de l'intégration sur le terrain, reste posée la question de la distanciation. Et lorsque l'on fonctionne dans la même langue, elle n'est pas évidente. II me faut parfois les remarques de mes collègues (« mais qu'est-ce que veut dire ce terme pour toi ? ») pour réaliser à quel point j'emploie dans mon vocabulaire quotidien des mots et des expressions du terrain.

Par cet apprentissage d'une langue tout à la fois différente de la mienne et tellement semblable, qui avec une syntaxe et des mots identiques signifie le monde particulier des producteurs de musique et les sépare du reste de la société, j'ai appris à connaître la culture. Qu'est-ce qu'apprendre à " parler métier », sinon faire "l'expérience de l'autre dans le même » (Augé 1989) ? [38] 
Daphy Éliane, 1994, "L'ethnologue dans le showbiz. Apprendre à parler métier ", Journal des anthropologues 57-58 (L'anthropologue face à la langue, coordonné par Diana Rey-Hulman et Galina Kabakova) : 29-39.

\section{Bibliographie}

AUGÉ Marc 1989, "L'autre proche », Segalen Martine (ed), L'autre et le semblable. Regards sur l'ethnologie des sociétés contemporaines, Paris, CNRS : 19-33.

DAPHY Eliane \& RAVEYRE Marie-Françoise 1988, « Show-bizz, la grande famille des gens du métier », Pour 112 "Formation et qualification : modes d'emploi » : 27-30 1989, « Nous on est du métier, à propos des représentations du travail », Segalen Martine, Michelat Claude \& Coadou Marie-Anne (eds), Anthropologie sociale et Ethnologie de la France, Actes du colloque du Centre d'Ethnologie Française et du Musée National des A.T.P, novembre 1987, Louvain-La-Neuve, Peeters : 677-686.

DEVEREUX Georges

1986, De l'angoisse à la méthode dans les sciences du comportement, Paris, Flammarion

GODELIER Maurice

1984, L'idéel et le matériel. Pensées, économies, sociétés, Paris, Fayard

LEVI-STRAUSS Claude

1962, La pensée sauvage, Paris, Plon

ROUSSIN Didier, JUTEAU Madeleine, BOUCHAUX Alain

1992, L'argot des musiciens, Castelnau-le-Letz, Climats

VAN GENNEP Arnold

1968 [1908], "Essai d'une théorie des langues spéciales », Revue des Etudes ethnologiques et sociologiques de Paris, Republications PAULET $1: 1-11$ 
Daphy Éliane, 1994, "L'ethnologue dans le showbiz. Apprendre à parler

métier », Journal des anthropologues 57-58 (L'anthropologue face à la

langue, coordonné par Diana Rey-Hulman et Galina Kabakova) : 29-39.

Journal des anthropologues Numéros 57 - 58 Automne-hiver 1994

[L'ANTHROPOLOGIE FACE A LA LANGUE]

ISSN 1156-0428

Notice Sudoc

http://corail.sudoc.abes.fr:80/DB=2.1/SET=6/TTL=1/CLK?IKT=1016\&TRM=L'anthropolog

ue + face $+\% E 1 a+l a+l a n g u e$

-Sommaire-

*Galina KABAKOVA, Diana REY-HULMAN

L'anthropologie et la linguistique : séparation de corps?

*Yves MONINO, Diana REY-HULMAN

Entretien avec Geneviève Calame-Griaule

*Eliane DAPHY

L'ethnologue dans le showbiz. Apprendre à parler métier

*Denis DECOURCHELLE

Ethnologie et surdité ou ce que l'on entend par langage

*Chantal DELTENRE-DE BRUYCKER

La bouche du village

*Sylvette DENEFLE

De la lexicométrie en anthropologie

*Marie-Paule FERRY

La linguistique : pourquoi faire?

*Eric GALLIBOUR

Le rapport de l'ethnologue à la langue de son terrain : l'effet miroir

*Galina KABAKOVA

L'ethnolinguistique en Russie : l'anthropologie aux mains des philologues

*Paulette ROULON-DOKO

L'anthropologue et la langue d'enquête

*Jean-Louis SIRAN

Les énoncés ne sont pas des choses, mais des événements

Revue de l'Association française des anthropologues (AFA)

Maison des Sciences de l'Homme - (Bureau 331)

54, bd Raspail - 75006 PARIS

Tel : 0149542181 - Emel : afa@msh-paris.fr 